\title{
Metástasis en el lóbulo frontal como primera manifestación clínica de un carcinoma folicular de tiroides
}

\author{
Frontal lobe metastases as the initial presentation of follicular thyroid carcinoma \\ Delia Luján-Martínez*, Miguel Ruiz-Marín, Emilio Peña-Ros, Antonio Albarracín-Marín-Blázquez y \\ Marifé Candel-Arenas \\ Servicio de Cirugía General y del Aparato Digestivo, Hospital General Universitario Reina Sofía, Murcia, España
}

\begin{abstract}
Resumen
Antecedentes: El carcinoma folicular de tiroides puede producir metástasis a distancia, siendo las localizaciones más habituales el pulmón y el hueso. Las metástasis cerebrales son inusuales, y es poco habitual que aparezcan como primera manifestación de cáncer. Caso clínico: Paciente varón de 80 años, que tras una caída al suelo comenzó con alteración del comportamiento, desorientación y pérdida del control de esfínteres. La tomografía computarizada cerebral evidenció una masa en el lóbulo frontal derecho. Se realizó la extirpación de la lesión cerebral, cuyo estudio histológico informó de metástasis de carcinoma folicular de tiroides. Conclusión: La manifestación de la enfermedad con metástasis cerebrales es muy infrecuente, y empeora el pronóstico notablemente.
\end{abstract}

PALABRAS CLAVE: Metástasis cerebral. Carcinoma folicular de tiroides. Carcinoma de tiroides.

\begin{abstract}
Background: Follicular thyroid carcinoma can produce distant metastases, generally occur to lung followed by bone. Brain metastases are unusual, and onset manifestation is little frequently. Clinical case: A 80-years old men presented disturbance behavior, desorientation and loss of sphincters after fallin to the ground. Computed tomography scan showed a lesion in the right frontal lobe. The patient underwent a craniotomy to remove the brain tumor, histopathology confirmed of follicular thyroid carcinoma metastases. Conclusion: At initial diagnosis brain metastasis are extremely rare, with adverse prognosis.
\end{abstract}

KEY WORDS: Brain metastases. Follicular thyroid carcinoma. Thyroid carcinoma.

\section{Antecedentes}

El carcinoma diferenciado de tiroides (CDT), incluyendo el carcinoma papilar (CP) y el carcinoma folicular (CF), se asocia a un pronóstico favorable, con una supervivencia a los 10 años del $80-90 \%$. Sin embargo, aunque son muy similares tienen un comportamiento diferente. El CP metastatiza con más frecuencia en los ganglios linfáticos regionales, y en no pocas ocasiones las adenopatías cervicales son el primer síntoma ${ }^{1}$. El CF está más vascularizado y, por tanto, produce metástasis a distancia con más frecuencia que el CP, siendo las localizaciones más habituales el pulmón y el hueso, pero la aparición de estas como primera manifestación es poco habitual. Las metástasis cerebrales (MC) de CDT son inusuales y la mayoría se diagnostican durante el seguimiento del tumor primario².

La presencia de MC empeora el pronóstico, ya que se considera como un estadio terminal de la enfermedad ${ }^{1}$.

\section{Correspondencia:}

*Delia Luján-Martínez

Avda. Intendente Jorge Palacios, $1 \quad$ Fecha de recepción: 04-03-2018

30003 Murcia, España

Fecha de aceptación: 28-07-2018

E-mail: deliamaria.lujan@gmail.com

DOI: $10.24875 / C I R U .18000207$

Cir Cir. 2018;86:562-565

Contents available at PubMed

www.cirugiaycirujanos.com 


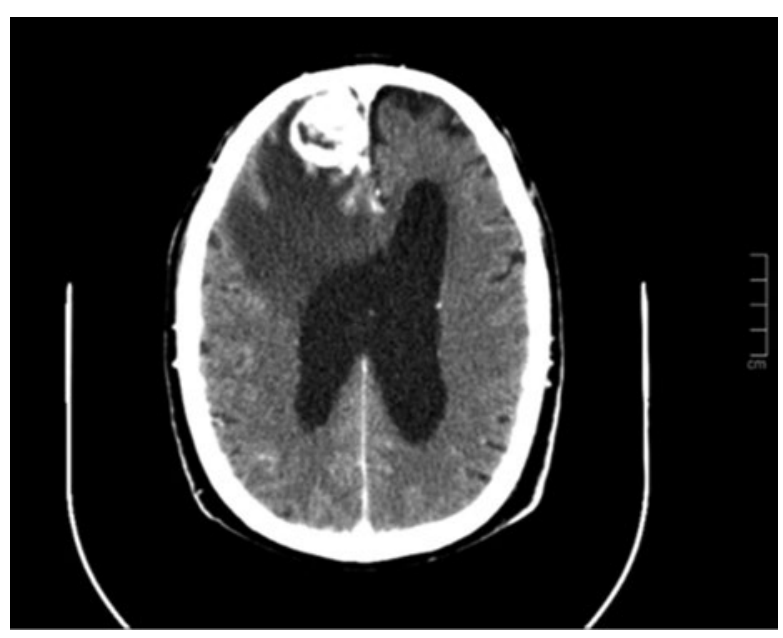

Figura 1. Tomografía computarizada cerebral que muestra una lesión de $29 \times 26 \times 30 \mathrm{~mm}$ en el lóbulo frontal derecho, con intenso realce heterogéneo tras la administración de contraste, áreas centrales necróticas y extensa alteración de la densidad adyacente por hipodensidad de morfología digitiforme en la sustancia blanca frontal y la rodilla del cuerpo calloso, con efecto masa sobre el sistema ventricular.
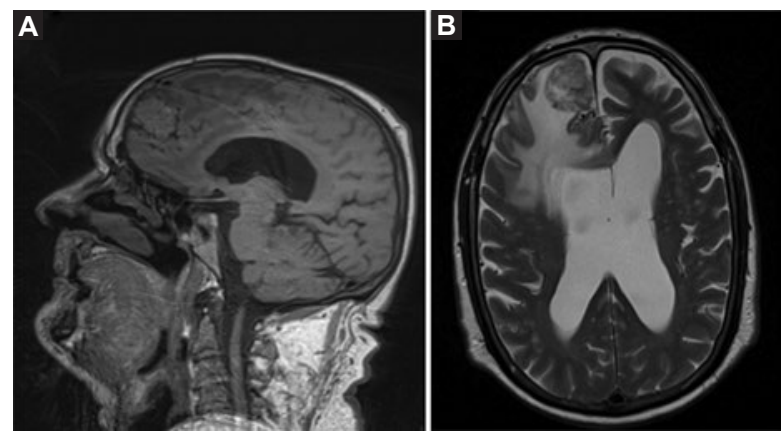

Figura 2. Resonancia magnética cerebral. A: corte sagital en T1 que muestra una masa frontal derecha situada en el giro frontal superior derecho, centrada en la unión corticosubcortical. B: corte axial en T2 en el que se observa una alteración de señal de la sustancia blanca adyacente que condiciona un importante efecto masa sobre los surcos y el sistema ventricular, y herniación subfalcial con un desplazamiento de la línea media de $8,5 \mathrm{~mm}$.

Presentamos un caso de CF de tiroides que tuvo como primera manifestación una metástasis cerebral en el lóbulo frontal.

\section{Caso clínico}

Varón de 80 años, sin antecedentes de interés excepto intervención en el oído izquierdo y fractura de fémur. Tras una caída al suelo comenzó con alteración del comportamiento, desorientación y pérdida del control de esfínteres. La exploración neurológica fue normal y en la tomografía computarizada cerebral (Fig. 1) se evidenció una masa de $29 \times 26 \times 30 \mathrm{~mm}$ en el lóbulo frontal derecho, con intenso realce heterogéneo tras la administración de contraste, áreas centrales necróticas y extensa alteración de densidad

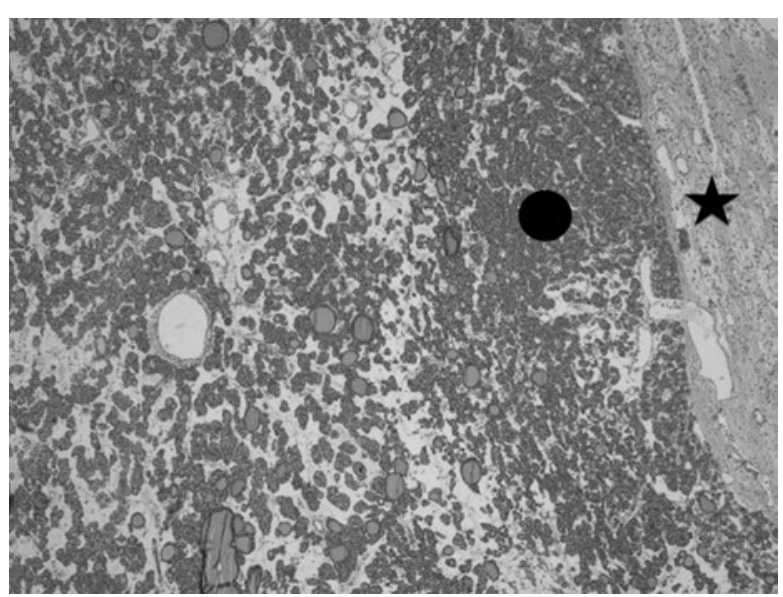

Figura 3. Hallazgos histológicos de invasión del parénquima cerebral (estrella negra) por células foliculares (círculo).

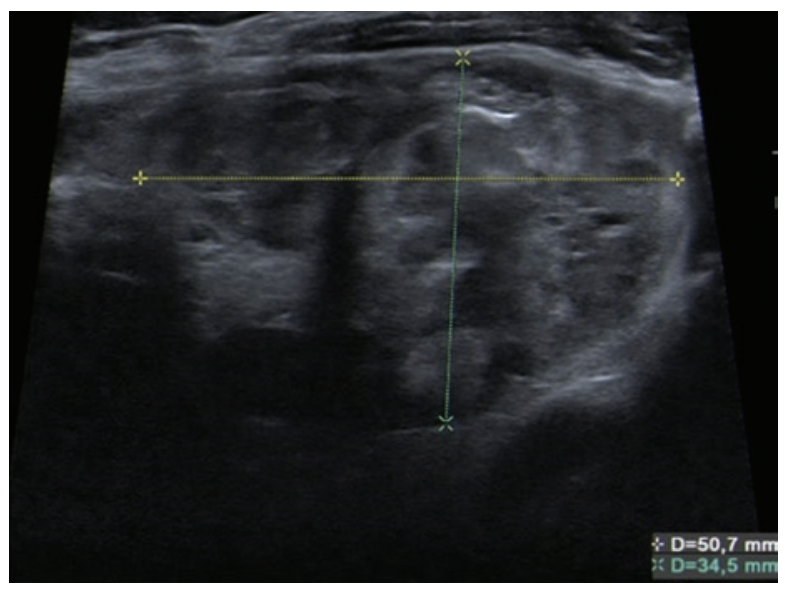

Figura 4. Ecografía cervical que muestra una lesión heterogénea en el lóbulo tiroideo izquierdo con calcificaciones en su interior.

adyacente por hipodensidad de morfología digitiforme en la sustancia blanca frontal y la rodilla del cuerpo calloso, con efecto masa sobre el sistema ventricular. La resonancia magnética (Fig. 2) objetivó contornos mal definidos en los márgenes posterior e inferior de la lesión, con extensa alteración de la señal en la sustancia blanca adyacente, de morfología digitiforme, que condiciona un importante efecto masa sobre los surcos y el sistema ventricular, con compresión del asta frontal y del cuerpo del ventrículo derecho, y signos de herniación subfalcial.

Con el diagnóstico de tumor primario de alto grado o metástasis única se intervino quirúrgicamente y se realizó la extirpación completa de la lesión cerebral, cuyo estudio histológico informó de metástasis de CF de tiroides (Fig. 3).

Se practicaron ecografía (Fig. 4) y tomografía computarizada cervicales, que evidenciaron una voluminosa masa en el lóbulo tiroideo izquierdo con calcificaciones y extensión intratorácica, con un tamaño aproximado 
de $5 \times 3,5 \mathrm{~cm}$, que contactaba con la vena yugular izquierda, deformándola, sin observar adenopatías. También se encontraron micronódulos pulmonares múltiples, en los lóbulos superiores, compatibles con metástasis. Se realizó punción-aspiración con aguja fina del nódulo, que informó de sospecha de CF. La analítica mostró una función tiroidea normal y una tiroglobulina muy elevada $(600,8 \mathrm{ng} / \mathrm{ml})$.

Se decidió realizar cirugía cervical y se practicó una tiroidectomía total bilateral al encontrar un nódulo duro y blanquecino, de más de $5 \mathrm{~cm}$, en el lóbulo tiroideo izquierdo. El posoperatorio cursó sin complicaciones. La anatomía patológica definitiva confirmó el diagnóstico de $\mathrm{CF}$ de tiroides. Actualmente, el paciente está en espera de recibir radioyodo.

\section{Discusión}

Las MC aparecen en el $20-30 \%$ de los pacientes con cáncer. Sin embargo, en los CDT ocurren en menos del $2 \%$ de los casos ${ }^{1}$. Se observan sobre todo en pacientes de edad avanzada, de sexo masculino, con tumor primario de grandes dimensiones y variedad histológica agresiva ${ }^{1-3}$, siendo inusual que sean la primera manifestación de la enfermedad. La localización más frecuente es en los hemisferios cerebrales ${ }^{4}$, como ocurrió en nuestro caso, aunque también pueden aparecer en el cerebelo y la pituitaria.

Clínicamente pueden cursar con cefalea, debilidad motora, pérdida visual y convulsiones, e incluso pueden ser asintomáticas ${ }^{5}$. La mayoría se diagnostican mediante técnicas de imagen, siendo las de mayor rendimiento la resonancia magnética y la tomografía computarizada. También pueden diagnosticarse al visualizar captación en el encéfalo al realizar el rastreo tras tratamiento $\operatorname{con}^{131} \mathrm{I}$. El intervalo medio entre el diagnóstico de CDT y la identificación de MC es de 2.9 años $^{6}$.

La presencia de metástasis pulmonares sincrónicas suele ser frecuente en el momento del diagnóstico ${ }^{2,3}$, aunque estas no son un factor predictivo significativo. Los pacientes con MC tienden a tener metástasis pulmonares cuando se diagnostican, como nuestro paciente.

Las concentraciones de tiroglobulina sérica se utilizan para detectar metástasis a distancia durante el seguimiento de los pacientes intervenidos de CDT'1 La tiroglobulina es un marcador tumoral que desciende tras aplicar el tratamiento adecuado, y si los valores se mantienen elevados podría indicar una recaída temprana de la enfermedad ${ }^{6}$. Los valores de la tiroglobulina están relacionados con la supervivencia global ${ }^{5}$. En nuestro caso se objetivaron valores elevados ya en el momento del diagnóstico ${ }^{1,5,6}$.

El curso clínico de las MC del CDT no está bien establecido, debido a su baja prevalencia. Estudios retrospectivos ${ }^{2,3}$ valoran factores pronóstico de supervivencia y concluyen que la edad ( $\leq 60$ años), tener menos de tres lesiones intracraneales y la ausencia de metástasis a distancia previas al desarrollo de MC se asocian de manera independiente con la supervivencia. En relación con estos datos, somos partidarios de realizar, siempre que sea posible, el tratamiento definitivo de las MC con el fin de mejorar la supervivencia.

El tratamiento de elección consiste en la resección quirúrgica de la MC seguida de radioterapia ${ }^{3,6,7}$. También debe considerarse la radioterapia en los pacientes que no puedan someterse a cirugía, y en caso de metástasis múltiples ${ }^{6}$. La resección completa del tumor primario asociado a tratamiento con radioyodo es fundamental ${ }^{8,9}$. En nuestro caso, el diagnóstico de CF se realizó tras la extirpación de la MC. Otros estudios ${ }^{10}$ sugieren que todas estas medidas terapéuticas no mejoran la supervivencia de los pacientes debido a la enfermedad sistémica avanzada, pero proporcionan un mejor control local del tumor cerebral metastásico.

En general, se acepta que los pacientes con MC de un CDT tienen una supervivencia más larga que la de aquellos con MC de otros tumores sólidos ${ }^{1}$. Para evitar las recidivas es fundamental que el tratamiento inicial del CF sea completo. Así mismo, consideramos que una vez diagnosticadas las MC, tanto en el seguimiento como si son la primera manifestación, es básica su resección completa seguida de radioterapia para prolongar la supervivencia.

\section{Responsabilidades éticas}

Protección de personas y animales. Los autores declaran que para esta investigación no se han realizado experimentos en seres humanos ni en animales.

Confidencialidad de los datos. Los autores declaran que han seguido los protocolos de su centro de trabajo sobre la publicación de datos de pacientes.

Derecho a la privacidad y consentimiento informado. Los autores declaran que en este artículo no aparecen datos de pacientes.

\section{Conflicto de intereses}

Los autores declaran que no existen conflictos de intereses. 


\section{Financiación}

Los autores declaran que no existe financiación externa.

\section{Bibliografía}

1. Lee HS, Yoo H, Lee SH, Gwak HS, Shin SH. Clinical characteristics and follow-up of intracranial metastases from thyroid cancer. Acta Neurochir. 2015;157:2185-94.

2. Saito F, Uruno T, Shibuya H, Kitagawa W, Nagahama M, Sugino K, et al. Prognosis after brain metastasis from differentiated thyroid carcinoma. World J Surg. 2016;40:574-81.

3. Choi J, Kim JW, Keum YS, Lee IJ. The largest known survival analysis of patients with brain metastasis from thyroid cancer base on prognostic groups. PLoS One. 2016;11:e0154739.
4. Pazaitou-Panayioutu K, Kaprara A, Chrisoulidou A, Boudina M, Georgiou E, Patakiouta F, et al. Cerebellar metastasis as first metastasis on papillary thyroid carcinoma. Endocr J. 2005;52:653-7.

5. Cacho-Díaz B, Spínola-Maroño H, Granados-García M, Reyes-Soto G, Cuevas-Ramos D, Herrera-Gómez A, et al. Metástasis cerebrales en pacientes con cáncer de tiroides. Med Int Mex. 2017;33:428-8.

6. Han E, Lee YY, Kim E, Kwon SY, Kim SP, Kim HS, et al. Solitary bone and brain metastasis in patient with papillary thyroid carcinoma mimicking cavernous angioma. Korean J Intern Med. 2016;31:991-4.

7. Ríos A, Galindo PJ, Rodríguez J, Tebar FJ, Parrilla P. Metástasis cerebral por un carcinoma folicular de tiroides. Tratamiento inicial inadecuado como factor pronóstico. Endocrinol Nutr. 2009:56:99-101.

8. Vrachimis A, Schmid KW, Jurgens $H$, Schober O, Weckesser M, Riemann B. Cerebral metastases from thyroid carcinoma: complete remission following radioiodine treatment. Dtsch Arztebl Int. 2013:110:861-6.

9. Durante C, Haddy N, Baudin E, Leboulleux S, Hartl D, Travagli JP, et al. Longterm outcome of 444 patients with distant metastases from papillary and follicular thyroid carcinoma: benefits and limits of radioiodine therapy. $\mathrm{J}$ Clin Endocrinol Metab. 2006;91:2892-9.

10. Tsuda K, Tsurushima H, Takano S, Tsuboi K, Matsumura A. Brain metastasis from papillary thyroid carcinomas. Mol Clin Oncol. 2013;1:817-9. 